\title{
Massive transfusion protocol: the reason it is necessary
}

\author{
DONG-KEUN KIM, YOUNG-HOON YOON, JUNG-YOUN KIM, YOUNG-DUCK CHO, SUNG-JUN PARK, SUNG-HYUK CHOI
}

Department of Emergency Medicine, Korea University College of Medicine, Seoul, Korea

Corresponding author:

Young-Hoon Yoon

Emergency Department,

Korea University Guro Hospital

Gurodong-ro 148, Guro-gu, Seoul, South Korea

Phone: +80-2-2626-1561, Fax: +80-2-2626-1562

E-mail:yyh71346@naver.com

\section{ABSTRACT}

Objective. The purpose of this study is to identify problems of emergency transfusion at the bedside and to determine need for massive transfusion protocol.

Methods. We included patients who met the criteria for "trauma team activation" and were admitted to division of trauma. The amount of blood product transfused in each unit was investigated for balanced transfusion. We also investigated the compliance with assessment of blood consumption score. The correlation between the time elapsed from patient visit to first transfusion order and time elapsed from first transfusion order to transfusion start was analyzed. Finally, we investigated various factors which serve to influence the decision-making process regarding early transfusion order.

Results. Ratio of packed Red blood cells (pRBC): Fresh frozen plasma (FFP) was well-balanced, but platelet transfusion done was much lower than $\mathrm{pRBC}$ and FFP in emergency room. The application of emergency blood release did not match the criteria of assessment of blood consumption $(\mathrm{ABC})$ score. The time from the first transfusion order to the transfusion start was found to be constant irrespective of time from patient visit to first transfusion order. And, the time from the first transfusion order to transfusion start did not differ significantly among patients with early transfusion order and delayed transfusion order. Only systolic blood pressure of $<90$ $\mathrm{mmHg}$ was identified as a major predictor for early transfusion order.

Conclusion. Balanced transfusion is not easy and emergency transfusion could be delayed at the bedside. Integrated and systematic structures for massive transfusion protocol would be invaluable and indispensable.

Keywords: transfusion, emergency, protocol

\section{INTRODUCTION}

Transfusion is one of the cornerstone therapies in the treatment of trauma patients. There are two important aspects of transfusion which improve trauma patient outcomes: rapid blood transfusion and balanced transfusion. Physical trauma typically results in death within six hours of the onset of hemorrhage. (1) The ideal resuscitation agent for traumatic shock is whole blood replacement because of its physiologic advantage. Otherwise, early co-administration of fresh frozen plasma (FFP) and platelets with packed red blood cell $(\mathrm{pRBC})$ could reduce morbidity and mortality. $(2,3)$

Recent military and civilian trauma data support the value of transfusion strategies; the implementation of a generally-accepted "transfusion strategy" would make it possible to deliver blood products more promptly. (4-8) The protocol for massive transfusions has proliferated worldwide since 2000, along with the concept of damage control resuscitation (DCR). In South Korea, the concept of massive transfusion protocol (MTP) has also become more prevalent in recent decades. There remain, however, some obstacles to clear up. The purpose of this study is to identify and investigate the specific problems associated with emergency blood release at the bedside focusing on the delayed and the imbalance of transfusion.

\section{MATERIALS AND METHODS}

\section{Study design and setting}

Retrospective chart reviews were conducted, pertaining to those patients admitted to the division of trauma via the emergency room (ER) at Korea University Guro Hospital (KUGH). KUGH is a 1050 bed hospital affiliated with a medical college. The emergency department (ED) of KUGH clocks approximately 60,000 patient visits per year. About twenty percent of them visit the $\mathrm{ED}$ due to trauma related injury. There are 40 regional emergency medical centers, nine trauma centers and three trauma surgeon training centers in South Korea. KUGH is a regional emergency medical center and includes a trauma surgeon training center. Trauma surgeons and emergency physicians of KUGH are all well acquainted with the MTP protocol and DCR, but there is, to date, no official massive transfusion protocol agreed upon among them. There is only an emergency blood release protocol in laboratory department. The emergency blood release protocol of $\mathrm{KUGH}$ aims to reduce pretransfusion test time to 15 minutes by only including $\mathrm{ABO}$ typing and saline phase of the cross-matching test. There are no ER refrigerators for uncross-matched type $\mathrm{O}$ blood in KUGH.

\section{Study Participants and Inclusion Criteria}

The study periods were from September 1, 2015 to August 31, 2017. During the designated study period, all of the patients who met the criteria for trauma team activation and were admitted to division of trauma via ER were included in this study. The criteria for trauma team activation in $\mathrm{KUGH}$ are as follows: systolic blood pressure $<90$ $\mathrm{mmHg}$, respiratory distress, initial Glasgow coma score $<12$ with truncal or extremity injury, unstable pelvic fracture, two or more proximal long bone fractures, degloving injury above elbow or knee joint level, penetrating injury to neck, chest or abdomen (including groin), fall from over 5 meters, simultaneous visit of 3 or more trauma patients at ER.

We excluded patients who did not receive a transfusion, received only platelet or FFP, patients younger than nineteen and patients transferred from other hospitals. 


\section{Date Sampling}

We collected patient data which included age, gender, mechanism of injury, injury diagnosis category, operation in 24 hours, initial vital sign at ER, amount of transfusion, the injury severity score (ISS), assessment of blood consumption (ABC) score and length of hospital stay. Two emergency specialists reviewed the chart. When the two chart reviewers were presented with conflicting data for continuous variables, the mean value of the above mentioned data was used. A third investigator reviewed the charts and determined which data was to be used in those cases which had conflicting data with categorical variables.

\section{Outcome measure}

The volume of blood product transfused in emergency room, intensive care unit, operation room and/or general ward was investigated. We also investigated the rate of emergency blood release activation depending on the $\mathrm{ABC}$ score. To find out whether rapid blood transfusion decisions can facilitate fast blood transfusion, the correlation between time elapsed from the first patient visit to the first transfusion order and time elapsed from first transfusion order to the actual transfusion start time was analyzed. Then, we divided the patients into two groups, one in which $\mathrm{pRBC}$ and FFP were ordered within 1 hour from ER visit (early transfusion order group) and the other in which $\mathrm{pRBC}$ and FFP were ordered over 1 hour from ER visit (delayed transfusion order group). Time from first transfusion order to transfusion start was compared between two groups. The time for first transfusion order was based on medical records entered in electronic charts. The transfusion start time was determined based upon documentation in the nursing chart in electronic form. KUGH utilizes an electronic hospital information system. In order to implement a doctor's prescription, a physician must directly access the computer system. Finally, we investigated various factors which serve to influence the decision-making process regarding early transfusion order.

\section{Statistical Analysis}

We conducted an independent $\mathrm{t}$ test to compare the mean value of the continuous variables and the Mann-Whitney test was used to compare continuous variables that did not show a normal distribution by Shapiro-Wilk test. The Kruskal-Wallis test

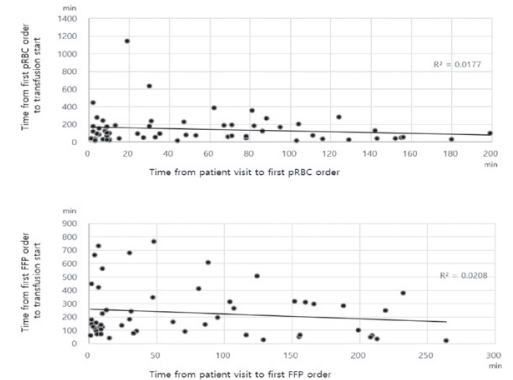

Figure 1. The correlation between transfusion order time and transfusion start time.

FFP, fresh frozen plasma; $p R B C$, packed $R B C$.

was used to compare mean value in three groups. A chi-squared analysis or Fisher's exact test was used for the categorical variables. Linear regression analysis was used to analyze correlation between transfusion order time and the actual transfusion time. Univariate logistic regression analysis was done to analyze the specific factors for early transfusion order. For the analysis of the univariate logistic regression analysis, we condensed injury mechanism into pedestrian-traffic accident or motorcycle or fall $>3 \mathrm{~m}$. The injury diagnosis category was condensed into chest, abdomen or pelvis. ISS $>45$, systolic blood pressure (SBP) < $90 \mathrm{mmHg}$, heart rate (HR) >120 and the result of focused assessment with sonography for trauma (FAST) were also investigated for logistic regression analysis. SPSS Statistics (SPSS 21.0, IBM, Chicago, USA) was used for statistical analysis.

\section{Ethics statement}

The present study protocol was reviewed and approved by the Institutional Review Board of Korea University Guro Hospital (approval No. KUGH 17071). Informed consent was waived by the board due to the retrospective nature of the study.

\section{RESULTS}

During the study period, a total of 64 eligible patients were identified in the database of the hospital information system. Three patients, whose time from patient visit to first transfusion order was over $400 \mathrm{~min}$ utes, were excluded. Finally, 61 patients were enrolled in this study.

\section{Baseline characteristics of the study population}

General data of included patients were

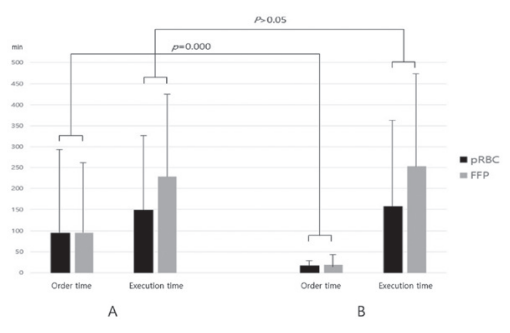

Figure 2. Time from first transfusion order to transfusion start in patients with early transfusion order (A) and delayed transfusion order (B).

FFP, fresh frozen plasma; pRBC, packed RBC.

shown in table 1 . The number of male patients was double that of female patients. The most common reported mechanisms of injury consisted of pedestrian-traffic accidents, and trips- or slips-and-falls. The two mechanisms noted above were found to comprise approximately half of injuries documented where treatment was sought. Pelvic bone fractures were the most common anatomical site in the injury diagnosis category. Thirty seven $(60.7 \%)$ patients underwent surgical intervention within 24 hours of the ER visit. The ratio of FFP frozen plasma and $\mathrm{pRBC}$ ratios was near $1: 1$, however, platelet transfusion rate was much lower. ISS was $43.8 \pm 12.3$ and ABC score was $0.9 \pm 0.9$ (Table1). The results of these scores show that included patients were severely injured and many of them needed emergency blood transfusions.

\section{Blood transfusion behavior analysis}

The largest volume of blood transfused occurred in the emergency rooms, followed by intensive care units, operating rooms and then general wards (Table 2). Table 2 also showed a well-balanced ratio of pRBC: FFP, but platelet transfusion was done much lower than $\mathrm{pRBC}$ and FFP in ER. Implementation of emergency blood release was done in thirteen out of fifteen patients $(86.7 \%)$ with $\mathrm{ABC}$ score of 2 or more. Thirty-five out of 46 people $(76.1 \%)$ with $\mathrm{ABC}$ score less than 2 were implemented with emergency blood release (Table 3).

\section{The relationship between blood transfu- sion and time factor}

Time form first transfusion order to transfusion start was constant irrespective of time from patient visit to first transfusion order as for $\mathrm{pRBC}$ and FFP (Figure 1). And time from first transfusion order to trans- 
Table 1. General characteristics of included patients

\begin{tabular}{|c|c|}
\hline Age (years) & $50.9 \pm 17.6$ \\
\hline \multicolumn{2}{|l|}{ Gender } \\
\hline Male & $40(65.6)$ \\
\hline Female & $21(34.49)$ \\
\hline \multicolumn{2}{|l|}{ Mechanism of injury } \\
\hline \multicolumn{2}{|l|}{ Traffic accident } \\
\hline In a vehicle & $6(9.8)$ \\
\hline Pedestrian & $17(27.9)$ \\
\hline Fall down & $15(24.6)$ \\
\hline Assault & $8(13.1)$ \\
\hline Bicycle & $3(4.9)$ \\
\hline Motorcycle & $4(6.6)$ \\
\hline Miscellaneous & $8(13.1)$ \\
\hline \multicolumn{2}{|c|}{ Injury diagnosis category } \\
\hline \multicolumn{2}{|l|}{ Abdomen } \\
\hline Kidney & $7(11.5)$ \\
\hline Liver & $11(18.0)$ \\
\hline Bowel & $21(34.4)$ \\
\hline Spleen & $10(16.4)$ \\
\hline \multicolumn{2}{|l|}{ Bone } \\
\hline Pelvis & $39(63.9)$ \\
\hline Long bone & $24(39.3)$ \\
\hline Spine & $8(13.1)$ \\
\hline Chest & $33(54.1)$ \\
\hline \multicolumn{2}{|l|}{ Operation in 24 hours } \\
\hline Operated & $37(60.7)$ \\
\hline Non-operated & $24(39.3)$ \\
\hline \multicolumn{2}{|l|}{ Initial vital sign } \\
\hline Initial SBP (mmHg) & $95.8 \pm 28.2$ \\
\hline $\mathrm{HR}$ (rate/min) & $92.6 \pm 22.4$ \\
\hline RR (rate/min) & $21.4 \pm 3.7$ \\
\hline $\mathrm{BT}\left({ }^{\circ} \mathrm{C}\right)$ & $36.3 \pm 0.8$ \\
\hline \multicolumn{2}{|c|}{ Amount of transfusion (unit) } \\
\hline $\mathrm{pRBC}$ & $5.0 \pm 4.4$ \\
\hline FFP & $4.3 \pm 4.5$ \\
\hline PLT & $1.3 \pm 3.4$ \\
\hline ISS & $43.8 \pm 12.3$ \\
\hline ABC score & $0.9 \pm 0.9$ \\
\hline \multicolumn{2}{|c|}{ Length of hospital stay (days) } \\
\hline Intensive care unit & $6.7 \pm 9.6$ \\
\hline General ward & $20.0 \pm 20.8$ \\
\hline Total stay & $26.7 \pm 25.9$ \\
\hline
\end{tabular}

Continuous variables are represented as mean \pm standard deviation. Categorical variables are represented as count (\%).

$\mathrm{ABC}$, assessment of blood consumption; BT, body temperature; FFP, fresh frozen plasma; HR, heart rate; ISS, injury severity score; PLT, platelet; pRBC, packed RBC; RR, respiration rate; SBP, systolic blood pressure.

Table 2. Amount of blood product transfused in emergency room, intensive care unit, operation room and general ward

\begin{tabular}{llllll}
\hline & No. of patients (\%) & ER & ICU & OP room & Ward \\
\hline pRBC & $61(100)$ & $3.8 \pm 3.1$ & $0.7 \pm 1.7$ & $0.3 \pm 1.1$ & $0.1 \pm 0.6$ \\
\hline FFP & $53(86.9)$ & $3.2 \pm 2.7$ & $0.8 \pm 2.4$ & $0.3 \pm 1.1$ & $0.1 \pm 0.4$ \\
\hline Platelet & $10(16.4)$ & $0.5 \pm 1.9$ & $0.8 \pm 2.5$ & $0.0 \pm 0.0$ & $0.1 \pm 0.5$ \\
\hline p-value & & 0.000 & 0.192 & 0.123 & 0.648 \\
\hline
\end{tabular}

FFP, fresh frozen plasma; pRBC, packed RBC. 
Table 3. Implementation of emergency blood release according to the assessment of blood consumption score

\begin{tabular}{lll}
\hline Emergency blood release & ABC score & \\
\hline & $>=2$ & $<2$ \\
\hline Yes & 13 & 35 \\
\hline No & 2 & 11 \\
\hline p-value & 0.360 & \\
\hline
\end{tabular}

Chi square: 0.360

$\mathrm{ABC}$, assessment of blood consumption; MTP, massive transfusion protocol.

Table 4. Logistic regression analyses of various variables for early transfusion order

\begin{tabular}{llll}
\hline Variables & Adjusted odd ratio & $\mathbf{9 5 \%}$ confidence interval & p value \\
\hline Age $>65$ years & 0.30 & $0.05-1.71$ & 0.18 \\
\hline Male & 1.32 & $0.31-5.64$ & 0.71 \\
\hline Body lesion & & & 0.17 \\
Chest & 0.30 & $0.05-1.68$ & 0.59 \\
Abdomen & 0.59 & $0.09-4.06$ & 0.79 \\
Pelvis & 0.79 & $0.14-4.57$ & 0.66 \\
\hline Mechanism of injury & & & 0.13 \\
Pedestrian TA, motorcycle injury & 0.66 & $0.11-4.10$ & 0.30 \\
Fall $>3$ m & 0.16 & $0.01-1.76$ & 0.00 \\
\hline ISS $>45$ & 2.51 & $0.44-14.42$ & 0.72 \\
\hline SBP $<90$ mmHg & 22.80 & $3.13-166.40$ & 0.92 \\
\hline HR $>120$ & 1.69 & $0.10-28.44$ & $0.19-4.52$ \\
\hline Positive FAST & 0.92 & & \\
\hline
\end{tabular}

$\mathrm{R} 2=0.443$

FAST, focused assessment with sonography for trauma; HR, heart rate; ISS, injury severity score; SBP, systolic blood pressure, TA, traffic accident.

fusion start was not different between in patients with early transfusion order and delayed transfusion order for pRBC (157.5 $\pm 210.7 \mathrm{~min}$ vs $148.5 \pm 176.8 \mathrm{~min})$ and FFP $(252.9 \pm 216.2 \mathrm{~min}$ vs $228.3 \pm 196.4 \mathrm{~min})$ (Figure 2).

\section{Predictors of early transfusion order in Trauma Patients}

Factors such as age ( $>65$ years), gender and the respective mechanisms of injury (as pedestrian-traffic accident or motorcycle or fall $>3 \mathrm{~m}$ ), the nature and extent of the trauma to the chest, abdomen or pelvis, ISS $>45, \mathrm{SBP}<90 \mathrm{mmHg}, \mathrm{HR}>120$ and the results of FAST were investigated for the predictors of early transfusion order (Table 4). Only SBP $<90 \mathrm{mmHg}$ was found to be the major and most reliable predictor for early transfusion order (odd ratio 22.80, 95\% confidence interval 3.13-166.40, $\mathrm{p}=0.00$ ).

\section{DISCUSSION}

Recent studies show that hemorrhage causes death at a rapid rate and takes a substantial number of patients at a fairly consistent rate among those patients with a history of physical trauma. (9) Trauma patients usually die due to hemorrhage within two hours, and $81 \%$ of all the trauma-related deaths occur within the initial 24-hour period. All are subsequently found to be hemorrhage-related. (10) As mentioned earlier in introduction, blood transfusion is one of the most critical therapies necessary to treat, or save, a seriously injured patient. In bleeding trauma patients, inadequate replacement of plasma and platelets may cause early death. Several studies have shown that providing appropriate blood transfusions early on can improve the outcomes of physicallyinjured patients. (11-13) During the last decade, studies of soldiers deployed to Iraq and Afghanistan who were wounded have demonstrated that a higher ratio of FFP to pRBC contributed to improve coagulation profile and reduced mortality, particularly when the treatment is provided within the window of time of the first six hours after injury. (14-16)

Although sufficient scientific evidence does not currently exist, most observational studies suggest a ratio of FFP and pRBC as 1:1. (17-22) Efficient transfusion protocol should be melded into hospital system to provide an appropriate ratio of $\mathrm{pRBC}$ and FFP with appropriate expedience and accuracy. Integrated and systematic structure is needed to provide early and consistent transfusion of blood products; it is one of the major components to be considered in planning multidisciplinary trauma systems.

For the immediate delivery of blood products and efficient resuscitation of severely injured patients, integrated and systematic protocols such as MTP have been adopted in many institutions. The implementation of MTP could reduce mortality, cost and organ failure (according to the results of several observational studies). These MTPs place emphasis on the standardization of coordination among the ED, laboratory staff and blood bank to ensure prompt delivery of blood products. (4,23-25)

Holcomb et al. pointed out that actual transfusion of blood product shows the unbalanced ratio of pRBC: FFP: platelet at the bedside. Especially, platelets transfused more lately than FFP. He explained that the unbalanced ratio of blood product transfu- 
sion was due to variations in blood product availability, absence of massive transfusion protocols and delays in blood bank to bedside transit times. (26)

As the result of Table 2, balanced transfusion is not easy in ER. Table 2 showed a well-balanced ratio of pRBC: FFP, but platelet transfusion was done much lower than $\mathrm{pRBC}$ and FFP. This finding can be explained as the following reason. KUGH does not stock platelets for transfusion within the hospital. When platelet transfusion is ordered, it must be transported from Korean Redcross Blood Services office, a 30-minute drive.

There is no official emergent transfusion protocol consensus within the inter department of KUGH. Table 3 shows that the application of emergency blood release did not match with the criteria of $\mathrm{ABC}$ score, one of the most common system for predicting MTP activation. (27) Only SBP $<90 \mathrm{mmHg}$ was the major predictor for early transfusion order in this study (Table 4). The others, even some of components for $\mathrm{ABC}$ score, did not draw a decision for emergency blood release. We suspect that this situation is due to the absence of a protocol for massive transfusion.

Figures 1 and 2 show the actual time re- quired to deliver blood product to bedside; the delivery might take anywhere from two to four hours, no matter how immediate or urgent the transfusion order. In theory, pre-transfusion test could be shortened to fifteen minutes by omitting part of the cross-matching test. For this reason, some physicians, believing that emergency blood release can be provided without delay to trauma patient in extremis, hesitate to transfuse blood type $\mathrm{O}$ for fear of an adverse transfusion reaction and prefer to provide matched blood product. Besides, there are many other factors that cause delays in the delivery of blood transfusion time at the bedside (delay in order, blood sampling, transport, etc.). The results of this study demonstrate the possibility of unanticipated delay in the delivery of needed blood product in the real world, unlike theory.

One of the major components of MTP protocols would be the placement of refrigerators in urgent care centers and emergency rooms for the purpose of un-cross matched type O blood. Most MTP address ER refrigerators for trauma patients with massive hemorrhage. Transfusion packs are also recommended with a target response time of 30 minutes when ordered.
However, as a result of Korean Survey for Emergency and Massive Transfusion Protocol, only one center (out of 40 regional emergency medical centers) and nine trauma centers have refrigeration for un-cross matched type O blood. (28)

There are some limitations to be considered in analyzing the results of this study. These study results were limited to a single hospital, which might generate a problem of faulty generalization. The other limitation is that this study design is a retrospective chart review. A more exact analysis could be done if before-and-after study was carried out. Lastly, we defined the included patients as those who met the criteria for trauma team activation and were admitted to division of trauma via ER. So, despite 2 years study periods, the number of study population was small.

To sum up, balanced transfusion is not easy and blood transfusion could be delayed in real aspect of bedside. Massive transfusion protocol including policy for un-cross matched type $\mathrm{O}$ blood would be invaluable and indispensable component for emergent transfusion.

\section{REFERENCES}

1. Demetriades D, Murray J, Charalambides K, Alo K, Velmahos G, Rhee P, et al. Trauma fatalities: time and location of hospital deaths. J Am Coll Surg 2004; 198(1): 20-6.

2. Borgman MA, Spinella PC, Perkins JG, Grathwohl KW, Repine T, Beekley AC, et al. The ratio of blood products transfused affects mortality in patients receiving massive transfusions at a combat support hospital. J Trauma 2007; 63: 805-13.

3. Pidcoke HF, Aden JK, Mora AG, Borgman MA, Spinella PC, Dubick MA, et al. Ten-year analysis of transfusion in Operation Iraqi Freedom and Operation Enduring Freedom: increased plasma and platelet use correlates with improved survival. J Trauma Acute Care Surg 2012; 73: S445-52.

4. Spinella PC, Holcomb JB. Resuscitation and transfusion principles for traumatic hemorrhagic shock. Blood Rev 2009; 23(6): 231-40.

5. Sinha R, Roxby D. Change in transfusion practice in massively bleeding patients. Transfus Apher Sci 2011; 45: 171-4.

6. O'Keeffe T, Refaai M, Tchorz K, Forestner JE, Sarode R. A massive transfusion protocol to decrease blood component use and costs. Arch Surg 2008; 143: 686-90.

7. Schuster KM, Davis KA, Lui FY, Maerz LL, Kaplan LJ. The status of massive transfusion protocols in United States trauma centers: massive transfusion or massive confusion? Transfusion 2010; 50: 1545-51.

8. Young PP, Cotton BA, Goodnough LT. Massive transfusion protocols for patients with substantial hemorrhage. Transfus Med Rev 2011; 25: 293-303.

9. Fox EE, Holcomb JB, Wade CE, Bulger EM, Tilley BC; PROPPR Study Group. Earlier endpoints are required for hemorrhagic shock trials among severely injured patients. Shock 2017; 47(5): 567-73.

10. Oyeniyi BT, Fox EE, Scerbo M, Tomasek JS, Wade CE, Holcomb JB. Trends in 1029 trauma deaths at a level 1 trauma center: Impact of a bleeding control bundle of care. Injury 2017; 48: 5-12.

11. Johnson JJ, Garwe T, Albrecht RM, Adeseye A, Bishop D, Fails RB, et al. Initial inferior vena cava diameter on computed tomographic scan independently predicts mortality in severely injured trauma patients. J Trauma Acute Care Surg 2013; 74: 741-5

12. Hagiwara A, Kushimoto S, Kato H, Sasaki J, Ogura H, Matsuoka T, et al. Can early aggressive administration of fresh frozen plasma improve outcomes in patients with severe blunt trauma?- a report by the Japanese Association for the Surgery of Trauma. Shock 2016; 45: 495-501.

13. Odell DD, Liao K. Superior vena cava and innominate vein reconstruction in thoracic malignancies: double-vein reconstruction. Semin Thorac Cardiovasc Surg 2011; 23: 326-9.

14. Baumann Kreuziger LM, Morton CT, Subramanian AT, Anderson CP, Dries DJ. Not only in trauma patients: hospital-wide implementation of a massive transfusion protocol. Transfus Med 2014; 24: 162-8.

15. Neal MD, Marsh A, Marino R, Kautza B, Raval JS, Forsythe RM, et al. Massive transfusion: an evidence-based review of recent de- 
velopments. Arch Surg 2012; 147: 563-71.

16. Phan HH, Wisner DH. Should we increase the ratio of plasma/platelets to red blood cells in massive transfusion: what is the evidence? Vox Sang 2010; 98: 395-402.

17. Elmer J, Wilcox SR, Raja AS. Massive transfusion in traumatic shock. J Emerg Med 2013; 44: 829-38.

18. Kashuk JL, Moore EE, Johnson JL, Haenel J, Wilson M, Moore JB, et al. Postinjury life threatening coagulopathy: is 1:1 fresh frozen plasma:packed red blood cells the answer? J Trauma 2008; 65(2):261-70; discussion 270-1

19. Gonzalez EA, Moore FA, Holcomb JB, Miller CC, Kozar RA, Todd SR, et al. Fresh frozen plasma should be given earlier to patients requiring massive transfusion. J Trauma 2007; 62: 112-9.

20. Gunter OL Jr, Au BK, Isbell JM, Mowery NT, Young PP, Cotton BA. Optimizing outcomes in damage control resuscitation: identifying blood product ratios associated with improved survival. J Trauma 2008; 65:527-34.

21. Dente CJ, Shaz BH, Nicholas JM, Harris RS, Wyrzykowski AD, Patel S, et al. Improvements in early mortality and coagulopathy are sustained better in patients with blunt trauma after institution of a massive transfusion protocol in a civilian level I trauma center. J Trauma 2009; 66: 1616-24.

22. Dzik WH, Blajchman MA, Fergusson D, Hameed M, Henry B, Kirkpatrick AW, et al. Clinical review: Canadian National Advisory Committee on Blood and Blood Products - massive transfusion consensus conference 2011: report of the panel. Crit Care 2011; 15(6): 242.

23. Cotton BA, Gunter OL, Isbell J, Au BK, Robertson AM, Morris JA Jr, et al. Damage control hematology: the impact of a trauma exsanguination protocol on survival and blood product utilization. J Trauma 2008; 64(5): 1177-82; discussion 1182-3.

24. Cotton BA1, Au BK, Nunez TC, Gunter OL, Robertson AM, Young PP. Predefined massive transfusion protocols are associated with a reduction in organ failure and postinjury complications. J Trauma 2009; 66: 41-8; discussion 48-9.

25.Zaydfudim V, Dutton WD, Feurer ID, Au BK, Pinson CW, Cotton BA. Exsanguination protocol improves survival after major hepatic trauma. Injury 2010; 41: 30-4.

26. Holcomb JB, del Junco DJ, Fox EE, Wade CE, Cohen MJ, Schreiber MA, et al, The prospective, observational, multicenter, major trauma transfusion (PROMMTT) study: comparative effectiveness of a time-varying treatment with competing risks. JAMA Surg 2013; 148(2): 127-36.

27. Nunez TC, Voskresensky IV, Dossett LA, Shinall R, Dutton WD, Cotton BA. Early prediction of massive transfusion in trauma: simple as ABC (assessment of blood consumption)? J Trauma 2009; 66(2): 346-52.

28. Cho D. Development of Standard Operation Manual for Emergency and Massive transfusion at Hospitals. Osong: Korea Centers for Disease Control and Prevention; 2017. 